\title{
Evaluation of IgG4 positive plasma cell infiltration in systemic plasmacytosis and other plasma cell infiltrating skin diseases
}

Shintaro Takeoka ${ }^{1}$, Masahiro Kamata ${ }^{1,2}$, Yayoi Tada ${ }^{1}$, Atsuko Fukuyasu ${ }^{1}$, Carren Sy Hau ${ }^{1}$, Takamitsu Ohnishi ${ }^{1}$, Yuko Sasajima ${ }^{3}$, and Shinichi Watanabe ${ }^{1}$

*1 Department of Dermatology, Teikyo University School of Medicine, 2-11-1 Kaga, Itabashi-ku, Tokyo 173-8605, Japan

*2 Department of Dermatology, University of Tokyo Graduate School of Medicine, 7-3-1 Hongo, Bunkyo-ku, Tokyo 113-8655, Japan.

*3 Department of Histopathology, Teikyo University School of Medicine, 2-11-1 Kaga, Itabashi-ku, Tokyo 173-8605, Japan
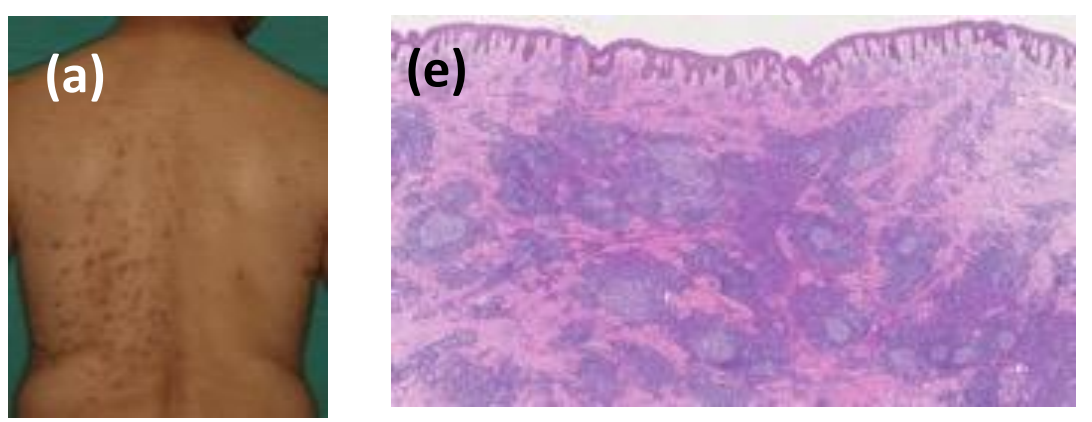

IgG
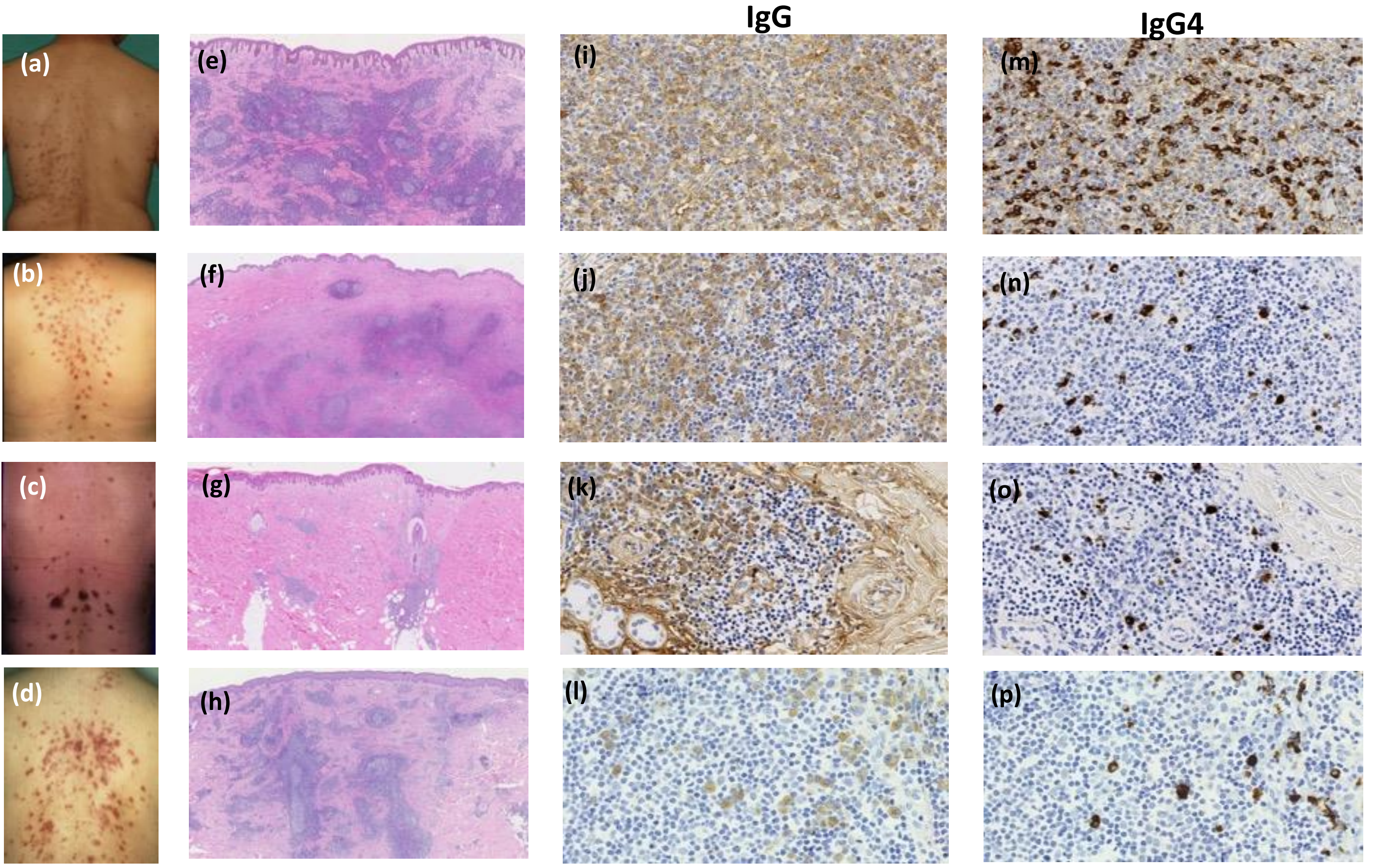

Figure Skin findings and histopathology of four cases of systemic plasmacytosis. (a-d) skin findings. (e-h) Hematoxylin and eosin staining ( $\times 40)$. (i-I) Immunostaining of IgG (×400). (m-p) Immunostaining of IgG4 (×400).

Background. IgG4 is a subclass of IgG, which is induced by Th2 cytokines such as interleukin (IL)-4 and IL-13, and has anti-inflammatory responses because of the unique structure. Systemic plasmacytosis (SP) is a rare skin disorder, which shows marked infiltration of plasma cells in the dermis. IgG4 related disease (IgG4-RD) is pathologically characterized by lymphoplasmacytic infiltration rich in IgG4-positive (IgG4+) plasma cells, storiform fibrosis, and obliterative phlebitis. It also demonstrates elevated serum IgG4 level. The relationship of SP and IgG4-RD has been recently argued.

Objective. To examine lgG4+/IgG+ plasma cell ratios in SP and other diseases which show marked infiltration of plasma cells.

Methods. The IgG4+/IgG+ plasma cell ratio was measured in four patients with SP and six patients with other plasma cell infiltrating diseases such as epidermal cyst, soft fibroma, systemic lupus erythematosus, cheilitis granulomatosa, squamous cell carcinoma, and IgG4-RD. Furthermore, we examined whether these cases met one of pathological features of comprehensive diagnostic criteria for IgG4-RD (over $40 \%$ ratio of IgG4+/IgG plasma cells).

Results. The results are shown in Figure and Table. Only one SP patient met the criteria (IgG4+/IgG+ plasma cell ratio \pm SD, the number of IgG4+ plasma cells per HPF \pm SD; $61 \pm 17 \%, 80.7 \pm 11$ [case 1]). However, three of four SP patients did not meet the criteria $(23 \pm 18 \%, 15 \pm 3.3$ [case 2], $9 \pm 9 \%, 7.7 \pm$ 3.9 [case 3], $29 \pm 11 \%, 34 \pm 4.9$ [case 4]. As for plasma cell infiltrating skin diseases other than SP, the specimen from chelitis granumatosa ( $73 \pm 27 \%$, 37 $\pm 5.1)$, SCC $(44 \pm 21 \%, 42.3 \pm 6.6)$ in addition to IgG4-RD $(91.9 \pm 4 \%, 129 \pm 29)$ met the IgG4-RD diagnosis criteria. Thus, three out of five chronic inflammatory skin diseases did not meet the IgG4-RD diagnosis criteria (Data not shown).

Discussion. Adding ten cases of previous reports together, six out of 14 patients (43\%) met one of pathological features of IgG4-RD diagnosis criteria (Table). None of our cases with SP showed storiform fibrosis or obliterative phlebitis even in the case with over $40 \%$ ratio of IgG4+/IgG+ plasma cells. Moreover, our study demonstrated that two out of five plasma cell infiltrating diseases other than SP showed over $40 \%$ ratio of IgG4+/IgG+ plasma cells. Strehl JD et al. reported that IgG4+ plasma cells are ubiquitous in non-specific chronic inflammatory conditions. Although the characteristic histopathological feature of abundant infiltration of IgG4+ plasma cells is essential for the diagnosis of IgG4-RD, abundant infiltration of IgG4+ plasma cells per se does not necessarily mean IgG4-RD. To diagnose IgG4-RD, clinical and other pathological manifestations should also be considered. These results suggest that $\mathrm{SP}$ is a distinct disease from IgG4-RD.

Table IgG4+/lgG+ plasma cell ratios of 14 SP patients

\begin{tabular}{|c|c|c|c|c|c|}
\hline Reference & Patient No. & Age (y) & Sex & Serum IgG4 (mg/dL) & IgG4+/IgG+ plasma cell (\%) \\
\hline \multirow{4}{*}{ Miyagawa-Hayashino A et al } & Case 1 & 54 & $\mathrm{M}$ & 610 & 42.2 \\
\hline & Case 2 & 55 & M & NA & 43.7 \\
\hline & Case 3 & 61 & $\mathrm{M}$ & NA & 58.1 \\
\hline & Case 4 & 46 & $M$ & 3040 & 50.1 \\
\hline \multirow{4}{*}{ Kato K et al } & Case 5 & 63 & M & NA & 33.0 \\
\hline & Case 6 & 38 & $M$ & 0 & 0 \\
\hline & Case 7 & 58 & $M$ & NA & 12.0 \\
\hline & Case 8 & 58 & $\mathrm{~F}$ & 56.9 & 22.4 \\
\hline Yamaguchi H et al & Case 9 & 63 & $M$ & 1250 & 54.0 \\
\hline Mizuno $\mathrm{M}$ et al & Case 10 & 60 & $M$ & 287 & 10.0 \\
\hline \multirow{4}{*}{ Our hospital } & Case 11 & 51 & $\mathrm{~F}$ & NA & 23.0 \\
\hline & Case 12 & 41 & $\mathrm{~F}$ & NA & 9.0 \\
\hline & Case 13 & 69 & $M$ & NA & 28.9 \\
\hline & Case 14 & 58 & $M$ & 120 & 61.1 \\
\hline
\end{tabular}

NOTE. IgG4, normal 408 to $105 \mathrm{mg} / \mathrm{dL}$; NA, not available; M, male; F, female 